\title{
Evaluación entre los miembros de la Sociedad Peruana de Odontopediatría en los criterios de tratamiento de la patología pulpar en dentición temporal
}

Perea M. Evaluación entre los miembros de la Sociedad Peruana de Odontopediatría en los criterios de tratamiento de la patología pulpar en dentición temporal. Rev Estomatol Herediana $2005 ; 15$ (2) : 145 - 149.

\section{Miguel Benjamín Perea Paz}

Docente del Departamento Académico de Estomatología del Niño y del Adolescente. Facultad de Estomatología. Universidad Peruana Cayetano Heredia.

\section{Correspondencia}

Miguel Perea Paz

Av. Honorio Delgado 430 Lima - 31, Perú

Teléfono: (51-1) 481-3380

e-mail: miguelpp@upch.edu.pe

\section{RESUMEN}

La terapia pulpar en las denticiones temporal y permanente joven es uno de los procedimientos más usados en odontopediatría. El propósito del presente estudio descriptivo fue evaluar los criterios de tratamiento de la patología pulpar entre los 120 miembros activos de la Sociedad Peruana de Odontopediatría (SPO). Se envió cartas con la descripción clínica y las radiografías de cinco casos clínicos de patología pulpar en dentición temporal. De 100 encuestas según años de ejercicio profesional, se encontró diferencias de criterios de evaluación de tratamiento de la patología pulpar: $32 \%$ obtuvo cuatro respuestas correctas y $26 \%$, tres respuestas correctas. Ninguno de los encuestados obtuvo respuesta deficiente. Los años de experiencia profesional, universidad de origen, asistencia a cursos, labor académica universitaria y estudios de postgrado no produjeron diferencias entre los criterios de evaluación para patología pulpar.

Palabras clave: PULPA DENTARIA. patología

Evaluation of members of the Peruvian Society of Pediatric Dentistry on treatment criteria of pulpar therapy in primary dentition

ABSTRACT

Pulpar therapy in temporary and permanent dentition is one of the most frequently used procedures in odontopediatrics. The purpose of this descriptive study is to identify the treatment criteria for pulpar pathology among 120 members of the Peruvian Society of Odontopediatrics (SPO). Letters were sent with the clinical descriptions and x-rays of five clinical cases of pulpar pathology in temporary dentition. In the 100 replies, differences in the evaluation criteria for pulpar pathology were detected. $32 \%$ of respondents had 4 correct answers and $26 \%$, three correct answers. None of the respondents had deficient answers. The years of experiences, school of origin, teaching experience or post-graduate studies produced no difference in the evaluation criteria for pulpar pathology.

Keywords: DENTAL PULP. pathology

\section{Introducción}

La terapia pulpar en dentición temporal y permanente joven es uno de los procedimientos más usados en odontología pediátrica. El objetivo principal es mantener los dientes temporales libres de patología pulpar hasta su exfoliación y promover al mismo tiempo el desarrollo radicular normal de las piezas permanentes jóvenes (1-3).

Para determinar el tipo de tratamiento que se va a seguir, es importante saber cuál es el estado de la pulpa dentaria y qué otros factores locales o sistémicos, pueden afectar el plan de tratamiento (4).

Es importante tener en cuenta que ningún signo o síntoma individual anticipa de manera absoluta la situación histopatológica de una pulpa afectada o del tratamiento $100 \%$ exitoso para la misma; debiendo obtenerse herramientas de diagnóstico, clínico y radiográfico (3).

Un problema adicional al diagnóstico y que lleva errores, es el difícil manejo del paciente en muchos casos (5).

En una revisión histórica Nunn (6) describió la evolución de los tratamientos pulpares y mencionó que Philip Pfaft en 1756, realizó el primer recubrimiento pulpar directo con pequeñas piezas de oro adaptadas cuidadosamente en la base de la cavidad; los progresos en el tratamiento de la patología pulpar de dientes temporales avanzaron con la tecnología y los nuevos materiales dentales. En 1826 Koeker propuso la cauterización de la exposición pulpar y luego cubrirla con laminillas metálicas. El primer medicamento en base al formaldehido que se tiene conocimiento fue utilizado por Nitzel en 8,000 casos.

En 1904 Buckley estudió el tratamiento de la pulpa necrótica y gangrenada utilizando una mezcla de partes iguales de tricresol y formalina dejando una torunda de algodón embebida del compuesto en forma hermética en la cámara pulpar por un día o una semana y posteriormente era removida en una segunda visita donde se colocaba el mismo compuesto en los conductos $(4,7,8)$.

Si fue Buckley el que introdujo el formocresol, fue Sweet en 1930 el que realizó ciertas modificaciones obteniendo hasta un $99,4 \%$ de éxito en pulpotomías y $88,1 \%$ en pulpectomías (9).

A mediados del presente siglo se re- portaron estudios donde se utilizaron hidróxido de calcio, glutaraldehido y formocresol. Sin embargo, el formocresol a través de su historia ha sido a menudo cuestionado por sus posibles efectos deletereos como carcinogenecidad, por lo que algunos autores han propuesto fórmulas alternativas en base al formaldehido $(7,8,10,11)$.

En nuestro país, la enseñanza del tratamiento de los problemas pulpares en dientes temporales está ocupando un espacio mayor en las currículas de la mayoría de las Facultades de Odontología, principalmente aquellas que cuentan con programas de segunda especialización. Sin embargo, las diferentes técnicas de terapia pulpar, las distintas escuelas dentales del país, así como también, las diferencias en el acceso a los cursos de actualización profesional hacen que la variabilidad de criterios respecto al tratamiento en casos con presencia de patología pulpar sea una preocupación (12).

Existen algunos estudios comparativos respecto a criterios de evaluación sobre el tratamiento de la patología pulpar en dientes temporales (5-13), sin embar- 
go ninguno de éstos se ha evaluado en el Perú, por lo que se desconoce el grado de uniformidad de criterios que existe para resolver un caso clínico de patología pulpar en forma adecuada.

El propósito del presente estudio es evaluar los criterios de tratamiento de la patología pulpar en dentición temporal entre los odontólogos que pertenecen a la Sociedad Peruana de Odontopediatría.

\section{Materiales y método}

La opinión de 120 miembros de la Sociedad Peruana de Odontopediatría sobre el tratamiento de la patología pulpar en piezas temporales fueron recogidos mediante un cuestionario con respuestas de múltiple elección, donde una sola alternativa era la correcta.

Criterios para elección de tratamientos en dentición temporal (según los casos presentados en la encuesta) tomados de la guía para la terapia pulpar en dentición temporal y permanente joven, American Academy of Pediatric Revisado (1998), fueron los siguientes:

1. Observación. Cuando la pieza temporal involucrada presenta cambio de color, no sintomatología ni evidencia de lesión radiolúcida en un examen radiográfico.

2. Exodoncia. Cuando la pieza presenta pérdida de función, lesión periapical amplia, compromiso de furcación, pocas posibilidades de restauración y cuando la pieza permanente está próxima a erupcionar (presencia de reabsorción radicular completa de las raíces de las piezas deciduas).

3. Pulpotomía. Procedimiento indicado cuando la pieza temporal presenta tejido coronal infectado y el remanente presenta diagnostico clínico y radiográfico de pulpa vital o afectada.

4. Pulpectomía. Está indicada cuando el diente temporal presenta exposición pulpar, y seguido de la amputación coronal, la pulpa radicular exhibe signos de hiperemia, como excesiva hemorragia o evidencia de necrosis pulpar con o sin lesión cariosa.

5. Restauración. Con ionómero, resina y amalgama

6. Recubrimiento Pulpar Indirecto. En dientes donde la lesión está próxima a la cámara pulpar. Un revestimiento protector o cemento puede ser colocado encima del remanente de la dentición lesionada para prevenir una exposición pulpar y estimular la cicatrización y reparación del tejido.
7. Recubrimiento Pulpar Directo. Este procedimiento es válido para exposiciones pulpares mecánica o traumáticas en dentición temporal cuando las condiciones para una respuesta favorable son óptimas. El procedimiento de recubrimiento pulpar por exposición pulpar por caries dental no es recomendable en dentición temporal.

Las respuestas fueron categorizadas de la siguiente manera :

5 respuestas acertadas $=$ optimo $(100 \%)$

4 respuestas acertadas $=$ bueno $(80 \%)$

3 respuestas acertadas $=$ aceptable $(60 \%)$

2 respuestas acertadas $=$ regular $(40 \%)$

1 respuesta acertada $=$ malo $(20 \%)$

0 respuesta acertada $=$ deficiente $(0 \%)$

Adicionalmente se evaluaron otras variables independientes realcionadas a la experiencia profesional de los miembros de la Sociedad Peruana de Odontopediatría tales como: años de experiencia profesional, universidad de procedencia, estudios de postgrado (mínimo un año), labor académico universitaria, $\mathrm{y}$ asistencia a cursos de la especialidad.

El análisis estadístico incluyó una prueba de chi cuadrado para determinar posible asociación entre las variables de estudio. La prueba de concordancia de Kendall's para determinar si las respuestas entre los distintos subgrupos estaban relacionadas, y las pruebas de Friedman y de correlación de Spearman.

\section{Resultados}

De las 120 encuestas enviadas a los miembros de la Sociedad Peruana de Odontopediatría se obtuvieron 100 respuestas.

La mayoría de los encuestados tuvo respuestas calificadas como buenas (32 encuestados, 32\%) y fue el subgrupo mayor a 21 años de ejercicio profesional, donde se presentó el mayor número (14 encuestados, 41\%) (Tabla 1). Es importante señalar que ningún encuestado obtuvo calificativo de deficiente.

Un total de 22 encuestados (22\%) obtuvieron el calificativo de óptimo, sobresaliendo el subgrupo de 6 a 10 años de ejercicio profesional con un total de 9 encuestados

En el grupo calificado como regular se encontraron 16 profesionales, ubicándose la mayoría de ellos en el subgrupos de 21 a más años de ejercicio profesional.

La prueba chi comprobó que el factor años de experiencia profesional no produce una diferencia estadísticamente significativa en los criterios de evaluación de tratamiento en los distintos subgrupos.

Las pruebas de Friedman y Kendall's demostraron que la correlación global fue estadísticamente significativa $(\mathrm{p}=0,032)$.

Según la Universidad de origen el mayor número de encuestados egresó de la Universidad Nacional Mayor de San Marcos (UNMSM) con 47 profesionales (47\%) seguida por la Universidad Peruana Cayetano Heredia (UPCH) con 24 (24\%), la Universidad Nacional Federico Villareal (UNFV) y la Universidad San Martín de Porres (USMP) con $8(8 \%)$ y $7(7 \%)$ profesionales respectivamente, la Universidad Nacional San Luis Gonzaga de Ica (UNSLG) con 5 (5\%) y la Universidad Católica Santa María de Arequipa (UCSM) con 3 (3\%). Procedente de Universidades del extranjero encontramos a $6(6 \%)$ profesionales (Tabla 2).

La mayoría de los encuestados egresados de la UNMSM, estuvieron agrupados dentro de los calificativos de buenas, aceptables y regulares. De los egresados de la UPCH, 8 obtuvieron calificación buena y 9 aceptables.

De acuerdo a la prueba de chi cuadrado se demostró que no existía asociación entre el factor Universidad de procedencia y los criterios de evaluación de tratamiento $(\mathrm{p}>0,05)$.

Al realizar el análisis de concordancia de Kendall's se encontró que existía una buena correlación entre los subgrupos procedentes de la UNMSM, con los de la UPCH $(0,55)$ y la UNSLG $(0,60)$.

Todos los profesionales asistían a cursos de actualización 80 de ellos (80\%) de manera frecuente y 20 sólo a veces (Tabla 3 ).

De los profesionales que asisten frecuentemente a los cursos, 26 (26\%) obtuvieron el calificativo de bueno, 22 fueron aceptables y $16(20 \%)$ obtuvieron el óptimo.

En el grupo que asiste sólo a veces a cursos, 6 obtuvieron el calificativo de óptimo y 6 el calificativo de bueno.

Del total de encuestados el 40\% (40 profesionales) ejerce labor docente. De estos 15 obtuvieron el calificativo de bueno, 12 aceptable y 9 el óptimo (Tabla 4).

Del total 57 (57\%) habían realizado estudios de post-grado (mínimo un año). De ellos 19 obtuvieron calificativo de aceptable, 18 bueno y 13 el óptimo (Tabla 5). 
Tabla 1. Evaluacion de los criterios de tratamiento según años de ejercicio profesional

\begin{tabular}{lcccccc}
\hline & \multicolumn{7}{c}{ Años de ejercicio profesional } \\
Calificación & $0-5$ & $6-10$ & $11-15$ & $16-20$ & 21 a más & total \\
\hline óptimo $(100 \%)$ & 5 & 9 & 2 & 1 & 5 & 22 \\
bueno $(80 \%)$ & 6 & 7 & 2 & 3 & 14 & 32 \\
aceptable $(60 \%)$ & 6 & 4 & 6 & 4 & 6 & 26 \\
regular $(40 \%)$ & 4 & 1 & 5 & 0 & 6 & 16 \\
malo $(20 \%)$ & 0 & 1 & 0 & 0 & 3 & 4 \\
deficiente $(0 \%)$ & 0 & 0 & 0 & 0 & 0 & 0 \\
\hline Total & 21 & 22 & 15 & 8 & 34 & 100 \\
\hline
\end{tabular}

Chi-cuadrado : $p>0,05 \quad$ Friedman: $p=0,032 \quad$ Kendall's: $p=0,032$

Tabla 2. Evaluación de los criterios de tratamiento según universidad de procedencia (pregrado)

\begin{tabular}{lccccccccc}
\hline \multirow{2}{*}{ Calificación } & UNMSM & UPCH & UNFV & UPSMP & UPGV & UNSLG & UNSM & Extranjero & total \\
\hline óptimo & 7 & 6 & 2 & 2 & 0 & 0 & 1 & 4 & 22 \\
bueno & 15 & 8 & 3 & 0 & 0 & 4 & 0 & 2 & 26 \\
aceptable & 10 & 9 & 1 & 4 & 0 & 1 & 1 & 0 & 24 \\
regular 1 & 2 & 1 & 2 & 1 & 0 & 0 & 0 & 0 & 16 \\
malo & 3 & 0 & 0 & 0 & 0 & 0 & 1 & 0 & 3 \\
deficiente & 0 & 0 & 0 & 0 & 0 & 0 & 0 & 0 & 0 \\
\hline Total & 47 & 24 & 8 & 7 & 0 & 5 & 3 & 6 & 100
\end{tabular}

UNMSM: Universidad Nacional Mayor de San Marcos. UPCH: Universidad Peruana Cayetano Heredia. UNFV: Universidad Nacional Federico Villareal. UPSMP: Universidad Particular San Martín de Porres. UPGV: Universidad Particular Garcilazo de la Vega. UNSLG: Universidad Nacional San Luis Gonzaga de Ica. UNSM: Universidad Nacional Santa María de Arequipa.

Chi-cuadrado: $p>0,05$ Kendall's : UNMSM-UPCH 0,55 buena correlación, UNMSM Vs. UNSLG 0,60 buena correlación

Tabla 3. Evaluación de criterios de tratameinto según asistencia a cursos de actualización en el área de odontopediatría

\begin{tabular}{lcccc}
\hline Calificación & \multicolumn{4}{c}{ Asistencia a cursos de actualización } \\
frecuentemente & a veces & no asiste & total \\
\hline óptimo & 16 & 6 & 0 & 22 \\
bueno & 26 & 6 & 0 & 32 \\
aceptable & 22 & 4 & 0 & 26 \\
regular & 12 & 4 & 0 & 16 \\
malo & 4 & 0 & 0 & 4 \\
deficiente & 0 & 0 & 0 & 0 \\
\hline Total & 80 & 20 & 0 & 100 \\
\hline
\end{tabular}

Chi-cuadrado : $p=1,000 \quad$ Friedman: $\mathrm{p}=0,75 \quad$ Kendall's: $\mathrm{p}=0,008$

Tabla 4. Evaluación criterios de tratamiento según labor académica universitaria

\begin{tabular}{lccc}
\hline & \multicolumn{3}{c}{ Ejercicio de labor docente } \\
Calificación & docente & no docente & total \\
\hline óptimo & 9 & 13 & 22 \\
bueno & 15 & 17 & 32 \\
aceptable & 12 & 14 & 26 \\
regular & 3 & 13 & 16 \\
malo & 1 & 3 & 4 \\
deficiente & 0 & 0 & 0 \\
\hline Total & 40 & 60 & 100 \\
\hline
\end{tabular}

Chi-cuadrado : $p=0,095 \quad$ Kendall's: $p=0,97 \quad$ Speraman $=0,000$
Tabla 5. Evaluación de criterios de tratamiento según estudios de postgrado en odontopediatría (mínimo un año)

\begin{tabular}{lccc}
\hline & \multicolumn{3}{c}{ Estudios de postgrado } \\
Calificación & postgrado & no postgrado & total \\
\hline óptimo & 13 & 9 & 22 \\
bueno & 18 & 14 & 32 \\
aceptable & 19 & 7 & 26 \\
regular & 5 & 11 & 16 \\
malo & 2 & 2 & 4 \\
deficiente & 0 & 0 & 0 \\
\hline Total & 57 & 43 & 100 \\
\hline Chi-cuadrado : $\mathrm{p}=1,0$ & Kendall's: $\mathrm{p}=0,47$ & Speraman $=0,19$
\end{tabular}




\section{Discusión}

El tratamiento pulpar en dientes temporales con caries con compromiso pulpar es el mejor servicio que puede recibir un niño para preservar sus piezas dentarias y por consiguiente el espacio para la dentición permanente (14).

Sin embargo a pesar de que hace muchos años se han descrito las técnicas de pulpotomía, pulpectomía y recubrimientos pulpares directos e indirectos en dentición temporal, en la actualidad los criterios de tratamiento de la patología pulpar en la primera dentición son muy variados y pueden depender de mucho factores como años de experiencia profesional, universidad de procedencia, así como el grado de actualización y especialización en Odontología Pediátrica (12).

Es importante resaltar que en la literatura revisada existen algunos estudios específicos sobre el tema como los de McKnight-Hanes et al. (12), Primosch et al. (13) y otros no específicos donde el método utilizado para poder investigar la opinión de tratamiento, de actividades y uso de materiales fue a través de encuestas enviadas y luego recibidas por correo convencional. Las limitaciones de este tipo de investigaciones es la tasa de repuesta, llamada tasa de retorno de la encuesta.

El presente estudio obtuvo una tasa de retorno fue de $83 \%$; porcentaje considerablemente más alto que los obtenidos por Kohli (15) (55\%), Seale y Kendrick (16) (43\%), Maestrello (17) (40\%) y McKnight-Hanes (12) (46\%), exceptuado la de Primosch (100\%), y esto puede deberse a la forma de envio y recepción de las encuestas.

Mientras que en las anteriores investigaciones, separando la de Primosch, la forma de envio y devolución de la encuesta fue por correo convencional, la actual investigación se entregó personalmente y se recogió de la misma forma por el mismo investigador, reforzando el método por llamadas telefónicas para coordinar la entrega.

Respecto a la opinión de tratamiento según años de ejercicio profesional, existen dos premisas; la primera es que los odontólogos egresados en los últi- mos años al estar más al día en conocimientos de nuevos tratamientos manejan mejor la opinión de tratamiento de patología pulpar y la segunda es que los odontólogos con más años de experiencia sean más acertados en el tratamiento de la patología pulpar.

Es importante destacar que la mayor información respecto al tema y además principal influencia es la Escuela Americana que con suma frecuencia realiza publicaciones en libros y revistas de la especialidad con los últimos avances en el campo de la patología pulpar (18). Sin embargo el estudio de Primosch, nos muestra que en los criterios los diferentes programas de postgrado en Estados Unidos existen algunas diferencias (13).

En el presente estudio, los resultados nos indican que el factor años de experiencia profesional, no produce una diferencia estadísticamente significativa en los criterios u opiniones de tratamientos en los grupos de edades que se crearon para esta investigación.

En los pocos estudios específicos del tema no evaluaron esta variable, pero McKnight-Hanes (12) concluye entre otras cosas que sería importante los años de experiencia profesional, como factor que podría explicar diferencias en la opinión de tratamiento de la patología pulpar.

Los criterios de tratamientos según universidad de procedencia en el presente estudio muestran que no existía asociación entre este factor y la opinión de tratamiento y MacKnight-Hanes (12) también recomienda que en futuros estudios la variable escuela o universidad de procedencia es otro factor que ayudaría a entender las diferencias de criterios. En esta investigación también se tomó en cuenta factores relacionados al grado de capacitación y actualización de los miembros de la Sociedad Peruana de Odontopediatría.

Respecto a la asistencia a cursos de actualización en el área de odontología pediátrica, ninguno de los encuestados manifestó no haber asistido a ningún curso y a pesar que los encuestados que asistieron frecuentemente a cursos de actualización es cuatro veces mayor que los que asisten sólo a veces, no se encontró asociación entre este factor y la opinión sobre el tratamiento.

Como información adicional respecto a este punto podemos mencionar que en el país y en especial en Lima, instituciones como las universidades, sociedades científicas en especial la Sociedad Peruana de Odontopediatría y los Colegios Odontológicos Departamentales frecuentemente dentro de su programación científica anual dictan cursos y conferencias sobre temas relacionados a la odontología pediátrica y en muchos casos específicamente del tema. Este resultado no es comparable con ninguno de otro estudio, puesto que no existen similares.

Como otro factor que podría ayudarnos a interpretar nuestros resultados fue referente a la labor docente, donde el $40 \%$ de los encuestados que devolvieron el cuestionario eran docentes de las distintas facultades de odontología del Perú. Como en casos anteriores el resultado de la prueba estadística utilizada nos mostró que no existía asociación entre el factor mencionado y los criterios de tratamiento usados.

Finalmente esta investigación también quería determinar la relación entre los encuestados que habian seguido estudios de postgrado (mínimo un año) y las respuestas y de la misma manera como en el resto de los factores no se encontró asociación.

McKnight-Hanes (12) en su investigación donde comparaba recomendaciones de tratamiento en dentición temporal entre dentistas generales y odontopediátras; encontró opiniones de tratamiento más acertadas entre los odontopediatras.

Comparándola con la presente investigación podríamos pensar que la muestra y distribución de la misma siendo mucho mayor que la nuestra, logró estadísticamente obtener mayores diferencias, Kholi et al. (15) en su investigación referente a la evaluación de anestésicos tópicos y anestesia local, concluyen que los odontopediatras manejan mejor el tema que los odontólogos de práctica general. 


\section{Conclusiones}

- Ningún encuestado obtuvo respuestas deficientes.

- Los años de experiencia profesional no produjeron diferencia de criterios de evaluación de la Patología Pulpar en dientes temporales entre los miembros activos de la Sociedad Peruana de Odontopediatría.

- No existe asociación entre la universidad de procedencia y los criterios de evaluación de tratamiento de la patología pulpar entre los miembros de la Sociedad Peruana de Odontopediatría.

- No se encontró asociación entre la asistencia a cursos de la especialidad y estudios de Postgrado en Odontopediatría con respecto a los criterios de evaluación de tratamiento de la Patología Pulpar.

- No existe asociación entre la labor académica universitaria del miembro de la Sociedad Peruana de Odontopediatría y los criterios de evaluación de tratamiento de la Patología Pulpar.

- No se halló asociación entre el factor estudios de postgrado y los criterios de evaluación de tratamiento de la Patología Pulpar.

\section{Agradecimiento}

A los miembros de la Sociedad Peruana de Odontopediatría que colaboraron en la realización del presente estudio.

\section{Referencias bibliográficas}

1. Castillo, R. Manual de Odontología Pediátrica. Caracas: Actualidades
Médico Odontológicas Latinoamérica; 1996.

2. Finn, S. Odontología Pediátrica. $4 \mathrm{a}$ ed. México, D.F: McGraw-Hill Interamericana; 1982.

3. Pinkham, JR. Odontología Pediátrica. 2a ed. México, D.F: McGraw-Hill Interamericana; 1991

4. Andlaw, R.J. Manual de Odontopediatría. 2a ed. México, D.F: McGraw-Hill Interamericana; 1999.

5. Guelmann M, Fair J, Turner C, Courts FJ. The success of emergency pulpotomies in primary molars. Pediatr Dent 2002; 24(3): 217-20.

6. Nunn JH, Smeaton I, Gilroy J. The development of formocresol as a medicament for primary molar pulpotomy procedures. ASDC J Dent Child 1996; 63(1): 51-3.

7. Judd PL, Kenny DJ. Formocresol concerns. A review. J Can Dent Assoc 1987; 53(5): 401-4.

8. Waterhouse PJ. Formocresol and alternative primary molar pulpotomy medicaments: a review. Endod Dent Traumatol 1995; 11(4): 157-62.

9. Roberts JF. Treatment of vital and non-vital primary molar teeth by one-stage formocresol pulpotomy: clinical success and effect upon age at exfoliation. Int J Paediatr Dent 1996; 6(2): 111-5.

10. Ranly DM, Horn D. Assessment of the systemic distribution and toxicity of formaldehyde following pulpotomy treatment: Part two. ASDC J Dent Child 1987; 54(1): 40-4.

11. Lewis B. Formaldehyde in dentistry: a review for the millennium. J Clin Pediatr Dent 1998; 22(2): 167-77.

12. McKnight-Hanes C, Myers DR, Dushku JC, Barenie JT. A comparison of general dentists' and pediatric dentists' treatment recommendations for primary teeth. Pediatr Dent 1991; 13(6): 344-8.

13. Primosch RE, Glomb TA, Jerrell RG. Primary tooth pulp therapy as taught in predoctoral pediatric dental programs in the United States. Pediatr Dent 1997; 19(2): 118-22.

14. Thompson KS, Seale NS, Nunn ME, Huff G. Alternative method of hemorrhage control in full strength formocresol pulpotomy. Pediatr Dent 2001;23(3): 217-22.

15. Kohli K, Ngan P, Crout R, Linscott CC. A survey of local and topical anesthesia use by pediatric dentists in the United States. Pediatr Dent 2001;23(3):265-9.

16. Seale NS, Kendrick AG. A survey of pediatric dentists' management of dental caries in children three years of age or younger. Pediatr Dent 2001; 23(3): 211-6.

17. Maestrello CL, Mourino AP, Farrington FH. Dentists' attitudes towards mouthguard protection. Pediatr Dent 1999; 21(6): 340-6.

18. Clinical Guideline on Pulp Therapy for Primary and Young Permanent Teeth. American Academy of Pediatric Dentistry. Pediatr Dent 2001; 23(7 Suppl): 58-61. 